International Journal of Applied Mathematics

Volume 32 No. $5 \quad 2019,775-784$

ISSN: 1311-1728 (printed version); ISSN: 1314-8060 (on-line version)

doi: http://dx.doi.org/10.12732/ijam.v32i5.5

\title{
SOME RESULTS ON UNIVALENT HOLOMORPHIC FUNCTIONS BASED ON $q$-ANALOGUE OF NOOR OPERATOR
}

\author{
Shahram Najafzadeh \\ Department of Mathematics \\ Payame Noor University \\ P.O. Box: 19395-3697, Tehran, IRAN
}

\begin{abstract}
The main object of this paper is to define a new sublclass of univalent holomorphic functions along with the recently defined $q$-analogue of Noor operator. We obtained a number of useful properties such as: coefficient bounds, extreme points, radii of starlikeness, convexity and close-to-convexity and weighted mean.
\end{abstract}

AMS Subject Classification: $30 \mathrm{C} 45,30 \mathrm{C} 50$

Key Words: univalent function, convolution, of Noor operator, coefficient estimate, convex set, extreme point, radii properties

\section{Preliminaries}

Let $\mathcal{A}$ be the class of all functions $f(z)$ which are analytic in $\mathbb{U}=\{z \in \mathbb{C}:|z|<$ $1\}$ and have the following Taylor series representation:

$$
f(z)=z+\sum_{k=2}^{\infty} a_{k} z^{k}
$$

Let us denote by $\mathcal{T}$ the subclass of $\mathcal{A}$ consisting of functions with negative coefficients of the form

$$
f(z)=z-\sum_{k=2}^{\infty} a_{k} z^{k} \quad\left(a_{k} \geqslant 0\right) .
$$

For functions $f$ and $g$ which are analytic in $\mathbb{U}$ and have the form (2), we define

Received: July 10, 2019

(C) 2019 Academic Publications 
the convolution (or Hadamard product) of $f$ and $g$ by:

$$
(f * g)(z)=z-\sum_{k=2}^{\infty} a_{k} b_{k} z^{k}, \quad(z \in \mathbb{U}) .
$$

Now, we provide some notations regarding the $q$-calculus used in this article, see $[1,3]$ and $[4]$.

For $0<q<1$, the $q$-derivative of $f$ is defined by:

$$
D_{q} f(z)=\frac{f(z q)-f(z)}{z(q-1)} \quad(z \neq 0) .
$$

We can easily conclude that:

$$
D_{q}\left(\sum_{k=2}^{\infty} a_{k} z^{k}\right)=\sum_{k=2}^{\infty}[k, q] a_{z} z^{k-1} \quad(k \in \mathbb{N}, \quad z \in \mathbb{U}),
$$

where

$$
[k, q]=\frac{1-q^{k}}{1-q}=1+\sum_{t=1}^{k-1} q^{t} \quad([0, q]=0),
$$

and

$$
[k, q]= \begin{cases}1 & , \quad k=0, \\ {[1, q][2, q] \cdots[k, q]} & , \quad k \in \mathbb{N} .\end{cases}
$$

Also, the $q$-generalization of the Pochhammer symbol for $y>0$ is defined by:

$$
[y, q]_{k}= \begin{cases}{[y, q][y+1, q] \cdots[y+k-1, q],} & k \in \mathbb{N}, \\ 1, & k=0 .\end{cases}
$$

For $\mu>-1$ and $f(z) \in \mathcal{T}$, we consider the $q$-analogue of Noor integral operator as follows:

$$
\mathcal{N}_{q}^{\mu} f(z)=\mathcal{T}_{q, \mu+1}^{-1}(z) * f(z)=z-\sum_{k=2}^{\infty} \Psi_{k-1} a_{k} z^{k} \quad(z \in \mathbb{U}),
$$

where

$$
\mathcal{T}_{q, \mu+1}^{-1}(z) * \mathcal{T}_{q, \mu+1}(z)=z D_{q} f(z)
$$




$$
\mathcal{T}_{q, \mu+1}(z)=z-\sum_{k=2}^{\infty} \frac{[\mu+1, q]_{k-1}}{[k, q] !} z^{k}
$$

and

$$
\Psi_{k-1}=\frac{[k, q] !}{[\mu+1, q]_{k-1}}
$$

see $[2]$.

It is clear that $\mathcal{N}_{q}^{0} f(z)=z D_{q} f(z), \mathcal{N}_{q}^{1} f(z)=f(z)$ and

$$
\lim _{q \rightarrow 1^{-}} \mathcal{N}_{q}^{\mu} f(z)=z-\sum_{k=2}^{\infty} \frac{k !}{(\mu+1)_{k-1}} a_{k} z^{k}
$$

which is the familiar Noor integral operator, see [5] and [6].

For $0 \leqslant \alpha \leqslant 1$ and $0 \leqslant \beta<1$, the function $f(z) \in \mathcal{T}$ is in the class $\mathcal{N}_{q}^{\mu}(\alpha, \beta)$ if it satisfies:

$$
\operatorname{Re}\left\{\frac{z D_{q}\left(\mathcal{N}_{q}^{\mu}(\alpha, \beta)\right)+\alpha z^{2} D_{q}^{2}\left(\mathcal{N}_{q}^{\mu} f(z)\right)}{\alpha z D_{q}\left(\mathcal{N}_{q}^{\mu} f(z)\right)+(1-\alpha) \mathcal{N}_{q}^{\mu} f(z)}\right\}>\beta,
$$

where $D_{q}$ and $\mathcal{N}_{q}^{\mu}$ are defined in (4) and (9) respectively. Also $D_{q}^{2}\left(\mathcal{N}_{q}^{\mu} f(z)\right)$ means $D_{q}\left[D_{q}\left(\mathcal{N}_{q}^{\mu} f(z)\right)\right]$.

\section{Main results}

In this section, we obtain coefficient bounds for functions in the class $\mathcal{N}_{q}^{\mu}(\alpha, \beta)$ and show that this class is a convex set.

Theorem 1. $f(z) \in \mathcal{T}$ is in the class $\mathcal{N}_{q}^{\mu}(\alpha, \beta)$ if and only if:

$$
\sum_{k=2}^{\infty} \Psi_{k-1}([k, q](1+\alpha[k, q]-\alpha \beta)+\beta(1-\alpha)) \alpha_{k} \leqslant 1-\beta,
$$

where $\Psi_{k-1}$ and $[k, q]$ are given by (12) and (6), respectively.

Proof. By making use of (4) and (5), we obtain:

$$
D_{q}\left(\mathcal{N}_{q}^{\mu}(\alpha, \beta)\right)=1-\sum_{k=2}^{\infty}[k, q] \Psi_{k-1} a_{k} z^{k-1},
$$




$$
D_{q}^{2}\left(\mathcal{N}_{q}^{\mu} f(z)\right)=-\sum_{k=2}^{\infty}[k, q]^{2} \Psi_{k-1} a_{k} z^{k-2},
$$

where $[k, q]$ and $\Psi_{k-1}$ are defined in (6) and (12), respectively.

By replacing (16) and (17) in (14) we have:

$$
\operatorname{Re}\left\{\frac{z-\sum_{k=2}^{\infty}[k, q] \Psi_{k-1} a_{k} z^{k}-\sum_{k=2}^{\infty} \alpha[k, q]^{2} \Psi_{k-1} a_{k} z^{k}}{A}\right\}>\beta
$$

where

$$
\begin{aligned}
A & =\alpha z-\sum_{k=2}^{\infty} \alpha[k, q] \Psi_{k-1} a_{k} z^{k} \\
& +(1-\alpha) z-\sum_{k=2}^{\infty}(1-\alpha) \Psi_{k-1} a_{k} z^{k}
\end{aligned}
$$

or

$$
\operatorname{Re}\left\{\frac{z-\sum_{k=2}^{\infty}[k, q] \Psi_{k-1}(1+\alpha[k, q]) a_{k} z^{k}}{z-\sum_{k=2}^{\infty} \Psi_{k-1}(\alpha([k, q]-1)+1) a_{k} z^{k}}\right\}>\beta
$$

By choosing the values of $z$ on the real axis and then letting $z \rightarrow 1^{-}$through real values, we get:

$$
\begin{aligned}
& 1-\beta-\sum_{k=2}^{\infty}\left[[k, q] \Psi_{k-1}(1+\alpha[k, q])\right. \\
& \left.-\beta \Psi_{k-1}(\alpha([k, q]-1)+1)\right] a_{k} \geqslant 0,
\end{aligned}
$$

or

$$
\sum_{k=2}^{\infty} \Psi_{k-1}[[k, q](1+\alpha[k, q]-\alpha \beta)-\beta(1-\alpha)] a_{k} \leqslant 1-\beta .
$$

Conversely, suppose that (15) holds true. We will show that (14) is satisfies and so $f \in \mathcal{N}_{q}^{\mu}(\alpha, \beta)$. Using the fact that $\operatorname{Re}\{W\}>\beta$ if and only if $|W-(1-\beta)|<$ $|W-(1-\beta)|$, it is enough to show that:

$$
L=\left|\frac{z D_{q}\left(\mathcal{N}_{q}^{\mu} f(z)\right)+\alpha z^{2} D_{q}^{2}\left(\mathcal{N}_{q}^{\mu} f(z)\right)}{\alpha z D_{q}\left(\mathcal{N}_{q}^{\mu} f(z)\right)+(1-\alpha) \mathcal{N}_{q}^{\mu} f(z)}-1-\beta\right|
$$




$$
<\left|\frac{z D_{q}\left(\mathcal{N}_{q}^{\mu} f(z)\right)+\alpha z^{2} D_{q}^{2}\left(\mathcal{N}_{q}^{\mu} f(z)\right)}{\alpha z D_{q}\left(\mathcal{N}_{q}^{\mu} f(z)\right)+(1-\alpha) \mathcal{N}_{q}^{\mu} f(z)}+1-\beta\right|=R
$$

But, if $\alpha z D_{q}\left(\mathcal{N}_{q}^{\mu} f(z)\right)+(1-\alpha) \mathcal{N}_{q}^{\mu} f(z)=J$, then we have:

$$
L=\frac{1}{|J|}\left[z D_{q}\left(\mathcal{N}_{q}^{\mu} f(z)\right)+\alpha z^{2} D_{q}^{2}\left(\mathcal{N}_{q}^{\mu} f(z)\right)-(1+\beta) J\right] .
$$

By (16) and (17) we get:

$$
\begin{aligned}
L & =\frac{1}{|J|}\left[\beta z-\sum_{k=2}^{\infty} \Psi_{k-1}[[k, q](1+\alpha[k, q]+(1-\beta))\right. \\
& \left.+(1-\beta)(1-\alpha)] a_{k} z^{k}\right] \\
& <\frac{|z|}{|J|}\left[\beta+\sum_{k=2}^{\infty} \Psi_{k-1}[[k, q](1+\alpha[k, q]+(1-\beta))\right. \\
& \left.+(1-\beta)(1-\alpha) a_{k}|z|^{k-1}\right]
\end{aligned}
$$

and

$$
\begin{aligned}
R & =\frac{1}{|J|}\left|z D_{q}\left(\mathcal{N}_{q}^{\mu} f(z)\right)+\alpha z^{2} D_{q}^{2}\left(\mathcal{N}_{q}^{\mu} f(z)\right)+(1-\beta) J\right| \\
& =\frac{1}{|J|} \mid(2-\beta) z-\sum_{k=2}^{\infty} \Psi_{k-1}([k, q](1+\alpha[k, q]+(1-\beta)) \\
& +(1-\beta)(1-\alpha)) a_{k} z^{k} \mid \\
& \geqslant \frac{|z|}{|J|}\left[(2-\beta)-\sum_{k=2}^{\infty} \Psi_{k-1}([k, q](1+\alpha[k, q]+(1-\beta))\right. \\
& \left.+(1-\beta)(1-\alpha)) a_{k}|z|^{k-1}\right] .
\end{aligned}
$$

when $z \in \partial \mathbb{U}=\{z \in \mathbb{C}:|z|=1\}$, it is easy to verify that $R-L>0$, if (15) holds and so the proof is complete. 
Remark. The result (15) is sharp for the function $F(z)$ given by:

$$
F(z)=z-\frac{1-\beta}{\Psi_{1}([2, q][k, q](1+\alpha[k, q]-\alpha \beta)+\beta(1-\alpha))} z^{2},
$$

where $\Psi_{1}=\frac{[2, q] !}{[\mu+1, q]_{1}}$ and $[2, q]=\frac{1-q^{2}}{1-q}=1+q$.

Corollary 2. If $f(z) \in \mathcal{N}_{q}^{\mu}(\alpha, \beta)$, then for $k=1,2, \ldots$, we have:

$$
a_{k} \leqslant \frac{1-\beta}{\Psi_{k-1}([k, q](1+\alpha[k, q]-\alpha \beta)+\beta(1-\alpha))} .
$$

Theorem 3. $\mathcal{N}_{q}^{\mu}(\alpha, \beta)$ is a convex set.

Proof. We must show that, if the functions $f_{t}(z), t=1,2, \ldots, m$, be in the class $\mathcal{N}_{q}^{\mu}(\alpha, \beta)$, then the function $h(z)=\sum_{t=1}^{m} \lambda_{t} f_{t}(z)$ for $\lambda_{t}$ and $\sum_{t=1}^{m} \lambda_{t}=1$, is also in $\mathcal{N}_{q}^{\mu}(\alpha, \beta)$.

By definition of $h(z)$, we conclude:

$$
\begin{aligned}
h(z) & =\sum_{t=1}^{m} \lambda_{t}\left(z-\sum_{k=2}^{\infty} a_{k, t} z^{k}\right) \\
& =z-\sum_{k=2}^{\infty}\left(\sum_{t=1}^{m} \lambda_{t} a_{k, t}\right) z^{k}
\end{aligned}
$$

But from Theorem 1, we have:

$$
\begin{aligned}
& \sum_{k=2}^{\infty} \Psi_{k-1}([k, q](1+\alpha[k, q]-\alpha \beta)+\beta(1-\alpha))\left(\sum_{t=1}^{m} \lambda_{t} a_{k, t}\right) \\
& =\sum_{t=1}^{m} \lambda_{t}\left\{\sum_{k=2}^{\infty} \Psi_{k-1}([k, q](1+\alpha[k, q]-\alpha \beta)+\beta(1-\alpha)) a_{k, t}\right\} \\
& \leqslant \sum_{t=1}^{m} \lambda_{t}(1-\beta)=1-\beta,
\end{aligned}
$$

which completes the proof. 


\section{Extereme points and some properties of $\mathcal{N}_{q}^{\mu}(\alpha, \beta)$}

In the last section, we obtain extreme points of $\mathcal{N}_{q}^{\mu}(\alpha, \beta)$ and investigate some properties of the some class.

Theorem 4. Let $f_{1}(z)=z$ and

$$
f_{k}(z)=z-\frac{(1-\beta) z^{k}}{\Psi_{k-1}([k, q](1+\alpha[k, q]-\alpha \beta)+\beta(1-\alpha))},
$$

where $k=2,3, \ldots$ Then $f \in \mathcal{N}_{q}^{\mu}(\alpha, \beta)$ if and only if it can be expressed in the form $f(z)=\sum_{k=1}^{\infty} t_{k} f_{k}(z)$, where $t_{k} \geqslant 0$ and $\sum_{k=1}^{\infty} t_{k}=1$. In particular, the extreme points of $\mathcal{N}_{q}^{\mu}(\alpha, \beta)$ are functions $f_{1}(z)$ and $f_{k}(z)$, where $k=2,3, \ldots$

Proof. Let $f$ be expressed as in the above. This means that we can write:

$$
\begin{aligned}
f(z) & =\sum_{k=1}^{\infty} t_{k} f_{k}(z)=t_{1} f_{1}(z)+\sum_{k=2}^{\infty} t_{k} f_{k}(z) \\
& =t_{1} z+\sum_{k=2}^{\infty} t_{k} z \\
& -\sum_{k=2}^{\infty} \frac{(1-\beta) t_{k}}{\Psi_{k-1}([k, q](1+\alpha[k, q]-\alpha \beta)+\beta(1-\alpha))} z^{k} \\
& =z \sum_{k=1}^{\infty} t_{k}-\sum_{k=2}^{\infty} d_{k} z^{k},
\end{aligned}
$$

where

$$
d=\frac{(1-\beta) t_{k}}{\Psi_{k-1}([k, q](1+\alpha[k, q]-\alpha \beta)+\beta(1-\alpha))} .
$$

Therefor $f \in \mathcal{N}_{q}^{\mu}(\alpha, \beta)$ since by Theorem 1, we have:

$$
\begin{aligned}
& \sum_{k=2}^{\infty} \frac{\Psi_{k-1}([k, q](1+\alpha[k, q]-\alpha \beta)+\beta(1-\alpha))}{1-\beta} d_{k} \\
& =\sum_{k=2}^{\infty} t_{k}=1-t_{1}<1 .
\end{aligned}
$$

Conversely, suppose that $f \in \mathcal{N}_{q}^{\mu}(\alpha, \beta)$. Theny by $(19)$, for $k=2,3, \ldots$, we have:

$$
a_{k} \leqslant \frac{1-\beta}{\Psi_{k-1}([k, q](1+\alpha[k, q]-\alpha \beta)+\beta(1-\alpha))} .
$$


By putting

$$
t_{k}=\frac{\Psi_{k-1}([k, q](1+\alpha[k, q]-\alpha \beta)+\beta(1-\alpha))}{1-\beta}, \quad(k \geqslant 2),
$$

we have $t_{k} \geqslant 0$ and if $t_{1}=1-\sum_{k=2}^{\infty} t_{k}$, we get the required result. So the proof is complete.

Theorem 5. Let the function $f(z)$ by (2) be in the class $\mathcal{N}_{q}^{\mu}(\alpha, \beta)$, then:

1. $f(z)$ is starlike of order $\delta_{1}$ for $0 \leqslant \delta_{1}<1$ in $|z|<R_{1}$,

$$
R_{1}=\inf _{k}\left[\frac{B}{\left(k-\sigma_{1}\right)(1-\beta)}\right]^{\frac{1}{k-1}}
$$

where

$$
B=\left(1-\delta_{1}\right) \Psi_{k-1}([k, q](1+\alpha[k, q]-\alpha \beta)+\beta(1-\alpha)) .
$$

2. $f(z)$ is convex of order $\delta_{2}$ for $0 \leqslant \delta_{2}<1$ in $|z|<R_{2}$, where:

$$
R_{2}=\inf _{k}\left[\frac{C}{k\left(k-2 \delta_{2}\right)(1-\beta)}\right]^{\frac{1}{k-1}}
$$

where

$$
C=\left(1-\delta_{2}\right) \Psi_{k-1}([k, q](1+\alpha[k, q]-\alpha \beta)+\beta(1-\alpha)) .
$$

3. $f(z)$ is close-to-convex of order $\delta_{3}$ for $0 \leqslant \delta_{3}<1$ in $|z|<R_{3}$, where:

$$
R_{3}=\inf _{k}\left[\frac{D}{k(1-\beta)}\right]^{\frac{1}{k-1}}
$$

where

$$
D=\left(1-\delta_{3}\right) \Psi_{k-1}([k, q](1+\alpha[k, q]-\alpha \beta)+\beta(1-\alpha)) .
$$

Proof. To establish the required result, it is sufficient to prove that:

$$
\left|\frac{z f^{\prime}(z)}{f(z)}-1\right| \leqslant 1-\delta_{1}, \quad\left(|z| \leqslant R_{1}\right) .
$$


But

$$
\begin{aligned}
& \left|\frac{z f^{\prime}(z)}{f(z)}-1\right|=\left|\frac{z-\sum_{k=2}^{\infty} k a_{k} z^{k}}{z-\sum_{k=2}^{\infty} a_{k} z^{k}}-1\right| \\
& =\left|\frac{-\sum_{k=2}^{\infty}(k-1) a_{k} z^{k}}{z-\sum_{k=2}^{\infty} a_{k} z^{k}}\right| \leqslant \frac{\sum_{k=2}^{\infty}(k-1) a_{k}|z|^{k-1}}{1-\sum_{k=2}^{\infty} a_{k}|z|^{k-1}} \leqslant 1-\delta_{1} .
\end{aligned}
$$

Thus $\sum_{k=2}^{\infty}\left(\frac{k-\delta_{1}}{1-\delta_{0}}\right) a_{k}|z|^{k-1} \leqslant 1$.

Since $f(z) \in \mathcal{N}_{q}^{\mu}(\alpha, \beta)$, the last inequality holds, if:

$$
|z|^{k-1} \leqslant\left[\frac{D}{\left(k-\delta_{1}\right)(1-\beta)}\right],
$$

where

$$
D=\left(1-\delta_{1}\right) \Psi_{k-1}([k, q](1+\alpha[k, q]-\alpha \beta)+\beta(1-\alpha)) .
$$

In the last theorem, we investigate the weighted mean concept.

Theorem 6. If $f$ and $g$ belong to $\mathcal{N}_{q}^{\mu}(\alpha, \beta)$, then the weighted mean of $f$ and $g$ is also in the some class.

Proof. We have to prove that $h_{t}(z)=\left[\frac{(1-t) f(z)+(1+t) g(z)}{2}\right]$ is in the class $\mathcal{N}_{q}^{\mu}(\alpha, \beta)$.

Since $f(z)=z-\sum_{k=2}^{\infty} a_{k} z^{k}$ and $g(z)=\sum_{k=2}^{\infty} b_{k} z^{k}$, so:

$$
h_{t}(z)=z-\sum_{k=2}^{\infty}\left\{\frac{(1-t) a_{k}+(1+t) b_{k}}{2}\right\} z^{k} .
$$

To prove $h_{t}(z) \in \mathcal{N}_{q}^{\mu}(\alpha, \beta)$, by (15) we need to show that:

$$
\sum_{k=2}^{\infty} \frac{E}{2(1-\beta)}<1
$$

where

$$
E=\Psi_{k-1}([k, q](1+\alpha[k, q]-\alpha \beta)+\beta(1-\alpha))
$$




$$
\times\left[(1-t) a_{k}+(1-t) b_{k}\right]
$$

For this, we have:

$$
\begin{aligned}
F & =\sum_{k=2}^{\infty} \frac{E}{2(1-\beta)} \\
& =\frac{(1-t)}{2} \sum_{k=2}^{\infty} \frac{\Psi_{k-1}[k, q](1+\alpha[k, q]-\alpha \beta)+\beta(1-\alpha)}{1-\beta} a_{k} \\
& +\frac{(1+t)}{2} \sum_{k=2}^{\infty} \frac{\Psi_{k-1}[k, q](1+\alpha[k, q]-\alpha \beta)+\beta(1-\alpha)}{1-\beta} b_{k}
\end{aligned}
$$

and by (15), we have:

$$
F<\frac{(1-t)}{2}+\frac{(1+t)}{2}=1 .
$$

Hence the result follows.

\section{References}

[1] A. Aral, V. Gupta, and R. P. Agarwal, Applications of q-Calculus in Operator Theory, Springer, 2013.

[2] M. Arif, M. U. Haq, and J.-L. Liu, A subfamily of univalent functions associated with-analogue of Noor integral operator, J. of Function Spaces, 2018 (2018), Art. \# 3818915, 5 pp.

[3] H. Exton, q-Hypergeometric Functions and Applications, Horwood, 1983.

[4] G. Gasper, M. Rahman, and G. George, Basic Hypergeometric Series, Volume 96, Cambridge University Press, 2004.

[5] K. I. Noor, On new classes of integral operators. J. Natur. Geom., 16, No 1-2 (1999), 71-80.

[6] K. I. Noor and M. A. Noor, On integral operators, J. of Math. Anal. and Appl., 238, No 2 (1999), 341-352. 